\title{
Can tackle height influence head injury assessment risk in elite rugby union?
}

\author{
Gregory J. Tierney*, Ciaran K. Simms \\ Trinity Centre for Bioengineering, Trinity College Dublin, Ireland
}

\section{A R T I C L E I N F O}

\section{Article history:}

Received 9 January 2018

Received in revised form 19 April 2018

Accepted 8 May 2018

Available online $\mathrm{xxx}$

\section{Keywords:}

Concussion

Head impact

Tackling

Injury prevention

\begin{abstract}
A B S T R A C T
Objectives: Tackle height laws are an area of controversy in rugby union. It is reported that the tackler is at most risk of a Head Injury Assessment (HIA). Therefore, the aim of this study was to use match video evidence of tackles in elite level rugby union to examine the effect of tackle heights on HIA risk for the tackler.

Design: Qualitative observational case-control study.

Methods: Each HIA $(\mathrm{n}=74)$ and control tackle $(\mathrm{n}=965)$ was categorised based on tackle direction (frontor side-on), tackle type (arm, shoulder or smother) and tackle height (upper trunk, mid-trunk, lower trunk, upper leg or lower leg). The Relative Risk (RR), 95\% Confidence Interval (CI) and probability $(p)$ values were calculated for each tackle height.

Results: Intended primary contact at the upper trunk of the ball carrier had a greater propensity to result in a HIA for the tackler for front-on upper body shoulder tackles $(\mathrm{RR}=1.48 ; 95 \% \mathrm{CI}=1.16-1.90 ; \mathrm{p}<0.01)$ and side-on upper body smother tackles $(\mathrm{RR}=2.30 ; 95 \% \mathrm{CI}=1.82-2.92 ; \mathrm{p}<0.01)$. Intended primary contact at the upper leg of the ball carrier had a greater propensity to result in a HIA for the tackler for front-on $(\mathrm{RR}=2.60 ; 95 \% \mathrm{CI}=1.70-3.97 ; \mathrm{p}<0.01)$ and side-on $(\mathrm{RR}=3.34 ; 95 \% \mathrm{CI}=1.65-6.79 ; \mathrm{p}<0.01)$ lower body shoulder tackles.

Conclusions: To reduce tackler HIA risk, the results suggest tackling below the upper trunk for upper body tackles. The results also suggest tackling at the lower trunk for lower body tackles and avoiding the upper legs. Prevention strategies should place emphasis on tackling lower risk body regions such as the midand lower trunk.
\end{abstract}

(C) 2018 Sports Medicine Australia. Published by Elsevier Ltd. All rights reserved.

\section{Introduction}

Rugby union is a territorial sport characterised by frequent contact between opposing players and/or the ground. ${ }^{1,2}$ For the sixth consecutive season, concussion was the most commonly reported match injury for English Premiership rugby (incidence rate of $20.9 / 1000$ player hours, contributing to $22 \%$ of all match injuries during the 2016-17 season). ${ }^{3}$ Tackling is regarded as the most common cause of injury and concussion in rugby union. ${ }^{4,5}$ Correct technique in the tackle is essential for safe and successful tackle outcomes, ${ }^{6-8}$ whilst poor tackle technique is a reported risk factor for injury ${ }^{9,10}$ and direct head impact causation. ${ }^{2,11}$ This indicates the importance for concussion injury prevention strategies.

In rugby union, retrospective analyses of match video evidence have previously been used to identify injury ${ }^{1,9}$ and head

\footnotetext{
* Corresponding author.

E-mail address: gtierne@tcd.ie (G.J. Tierney).
}

impact/concussion risk factors ${ }^{2,11,12}$ as well as guide prevention techniques. ${ }^{2,9,11}$ Recent tackle technique studies ${ }^{2,13}$ have categorised legal tackles as either upper body or lower body tackles. An upper body tackle is defined by the tackler's intended initial contact being above the ball carrier's hip while a lower body tackle is defined as the tackler's intended initial contact being at or below the ball carrier's hip. ${ }^{2}$ One study ${ }^{2}$ found that tacklers were at most risk of sustaining a direct head impact and that upper body tackles were the greatest cause of this. However, the upper body and lower body tackle definitions utilised in this study covered a wide range of body regions. Therefore, the study reports limited information on specific tackle height analysis for direct head impact aetiology. In particular, this study did not conduct a direct head impact risk analysis for specific tackle heights. The tackle height law in rugby union is set at the line of the ball carrier's shoulder and any contact above this line is regarded as foul play. ${ }^{14}$ It has been an area of concern with respect to injury for many years. ${ }^{15}$ Lowering the maximum legal tackle height has been recommended since the $1970 \mathrm{~s},{ }^{15}$ but the evidence base for this is limited. To guide concussion prevention 
strategies and before tackle laws can be changed, it is essential to examine the effect of tackle heights on head impact and concussion risk.

The Head Injury Assessment (HIA) was introduced in 2012 by World Rugby as the pitch side assessment process for concussion injuries and has previously been described in detail. ${ }^{16}$ Briefly, the HIA provides a standardised tool for the medical assessment of concussion injuries in rugby. ${ }^{16}$ A player enters the HIA protocol by exhibiting on-field signs and symptoms of concussion. ${ }^{16}$ The HIA examines a range of concussive symptoms ${ }^{17}$ including both immediate and delayed memory difficulties, cognitive ability, balance and player discomfort. If a player does not pass the HIA, he or she is removed from play and must follow the return-toplay protocol. Players diagnosed with a concussion are managed through the the graduated return to play protocol set out by World Rugby. ${ }^{3}$ The protocol encompasses six stages which each player must complete sequentially: (1) physical and cognitive rest until asymptomatic; (2) light aerobic exercise; (3) sport specific exercise; (4) non-contact training drills; (5) full contact practice; (6) return to play. Players can only progress to the next stage if they remained asymptomatic for an unbroken period of $24 \mathrm{~h}^{3}{ }^{3}$ The minimum time to return to play is 6 days from the day of injury. ${ }^{3}$ It is clear that a reduction in tackle-related HIAs would be evidence of concussion injury reduction within rugby union. Accordingly, the aim of this study was to use match video evidence of tackles in elite level rugby union to examine the effect of tackle heights on HIA risk for the tackler (based on intended primary contact location on the ball carrier ${ }^{2}$ ), for both upper body and lower body tackles.

\section{Methods}

A qualitative observational case-control study design was used to identify the risks associated with specific tackle heights on HIA aetiology in men's professional rugby union using match video evidence. A tackle was defined as "when the ball-carrier was contacted (hit and/or held) by an opponent without reference to whether the ball-carrier went to ground". ${ }^{1}$ A HIA tackle was defined as "when a tackler received a direct/indirect head impact in the tackle and was subsequently removed from play for a HIA and did not return to play for the remainder of the game." ${ }^{11}$ All data was freely available online and no medical data on individuals is reported for this study. As a result of this, no ethical permission was required similar to other rugby union video analysis studies on head impacts ${ }^{2,18}$ and knee injuries. ${ }^{19}$ A non-HIA tackle was defined as "when a player did not receive an injury/head impact/HIA (including HIAs that resulted in temporary and permanent removal from the game) as a result of the tackle."

The HIA video data from Tierney et al. ${ }^{11}$ was utilised for this study. In brief this data consists of 74 tackles that resulted in a HIA for the tackler (19 upper body and 19 lower body for front-on tackles and 23 upper body and 13 lower body for side-on tackles) from elite level competitions including Pro 12 (2014-2017), European Rugby Champions Cup games (2014-2017), RBS 6 Nations (2014-2017), Guinness Autumn Test Series (2013-2016), Rugby World Cup warm-up games (2015), the Rugby World Cup (2015) and the British and Irish Lions Tour (2017). Although a HIA can occur from an impact to the body, ${ }^{17}$ a direct head impact was identified in every video. To provide non-HIA cases as a control cohort, the dataset from Tierney et al. ${ }^{20}$ was utilised. In brief, this dataset consisted of five randomly selected games from the abovementioned competitions. Every non-HIA tackle $(n=965)$ from these five games was analysed for the control cohort dataset. No tackles that resulted in a temporary HIA (player returned to play during the game) occurred in the five games of the control cohort.
Each tackle analysed was categorised based on tackle direction (front-or side-on), tackle type (arm, collision, jersey, lift, shoulder, smother or tap ${ }^{14}$ ) and tackle height (upper trunk, mid-trunk, lower trunk, upper leg or lower leg, see Fig. 1). Fuller et al. ${ }^{14}$ defined the following for arm, shoulder and smother tackles; Arm Tackle "Tackler impedes/stops ball carrier with upper limb(s)"; Shoulder tackle - "Tackler impedes/stops ball carrier with shoulder as the first point of contact followed by use of arm(s)"; Smother tackle "Tackler uses chest and wraps both arms around ball carrier". The following definitions were utilised for the tackle height analysis; Upper trunk-line of the shoulders to base of the chest/pectorals; Mid-trunk-base of chest/pectorals to top of pelvis; Lower trunk - top of pelvis to base of pelvis; Upper leg - base of pelvis to base of knees; Lower leg - below base of knees, see Fig. 1. One reviewer (Biomechanist) analysed each video. The videos were analysed using Sports Code (Version 8) enabling a frame-by-frame viewing of the tackle. The video had a minimum frame rate of $25 \mathrm{fps}$ and could be watched as many times as necessary.

Sixty tackles (including HIA and non-HIA cases) were randomly selected using a random number generator (http://www.random. $\operatorname{org} /$ ). The reviewer then conducted the analysis on these 60 cases, for each tackle variable (tackle height, direction and type), at least one week after conducting the initial set of cases. Intra-rater reliability was then assessed using Cohen's Kappa (K). To assess inter-rater reliability, an external reviewer (ex-player) conducted the analysis on the same 60 cases using the same protocol as the main reviewer. Similarly, inter-rater reliability was then assessed using Cohen's Kappa (K). A Cohen's Kappa value greater than 0.8 indicates almost perfect agreement. ${ }^{21}$ For intra-rater reliability, Cohen's kappa values of $0.93,0.97$ and 0.92 were achieved for tackle height, direction and type, respectively. For inter-rater reliability, Cohen's kappa values of $0.83,0.83$, and 0.82 were achieved for tackle height, direction and type, respectively.

For upper and lower body front- and side-on tackles, only the main tackle type that resulted in a HIA for each of these categories (see Fig. 2) and the tackle type matched control cases were utilised for the statistical analysis. The Relative Risk (RR), 95\% Confidence Interval $(\mathrm{CI})$ and probability $(p)$ values were calculated for each tackle height. 2,14 The RR for each height was calculated by comparing the frequency of occurrence for HIA cases with the frequency of occurrence in non-HIA cases. An RR $=1$ indicates that the tackle height has no greater propensity to cause a HIA than that anticipated by chance; an $R R>1$ and $R R<1$ indicates that the tackle height has a greater and lesser propensity to cause a HIA than that expected by chance, respectively. ${ }^{2,14}$ A variable was considered to have statistical significance if the $95 \% \mathrm{CI}$ for the RR value did not include 1 and the $p$-value was $<0.05$. The $95 \% \mathrm{CI}$ was also reported for the frequency of occurrence results. ${ }^{22} \mathrm{RR}$ values $>1$ and $<1.11, \geq 1.11$ and $<1.43, \geq 1.43$ and $<2.00, \geq 2.00$ and $<3.33, \geq 3.33$ and $<10$ were indicative of trivial, small, medium, large and very large, respectively. ${ }^{23}$ Similarly, RR values $<1$ and $>0.90, \leq 0.90$ and $>0.70$, $\leq 0.70$ and $>0.50, \leq 0.50$ and $>0.30, \leq 0.30$ and $>0.10$ were indicative of trivial, small, medium, large and very large, respectively. ${ }^{23}$

\section{Results}

Fig. 2 shows that shoulder (79\%; $n=15)$ and smother tackles $(65 \% ; n=15)$ account for the majority of upper body front- and side-on tackles, respectively. Also, shoulder tackles account for the majority of lower body front- and side-on tackles (95\%; $n=18$ and $71 \%$; $n=10$, respectively)

Table 1 shows that intended primary contact at the upper trunk of the ball carrier had a greater propensity to result in a HIA for the tackler for front-on upper body shoulder tackles $(\mathrm{RR}=1.48 ; 95 \%$ $\mathrm{CI}=1.16-1.90 ; \mathrm{p}<0.01)$ and side-on upper body smother tackles 
(a)

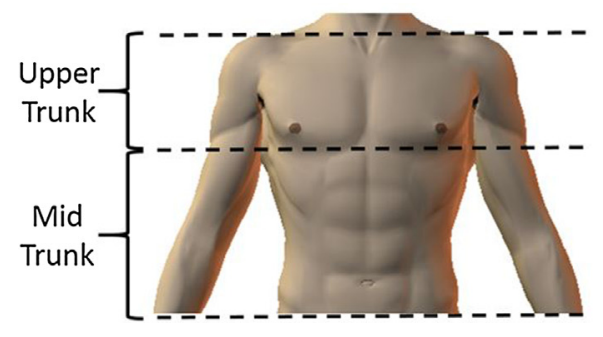

(b)

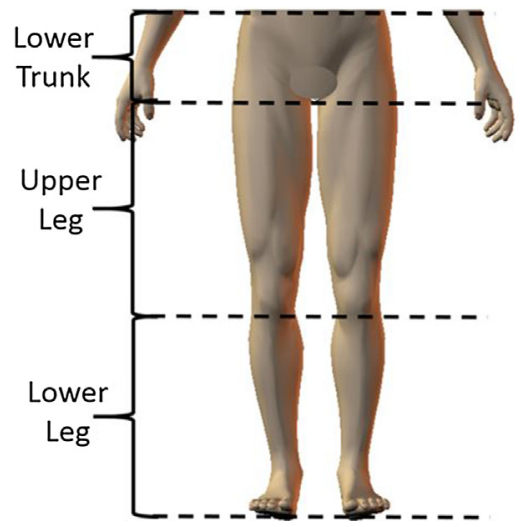

Fig. 1. The ball carrier's body split into (a) upper body and (b) lower body regions.

UBT Front-on

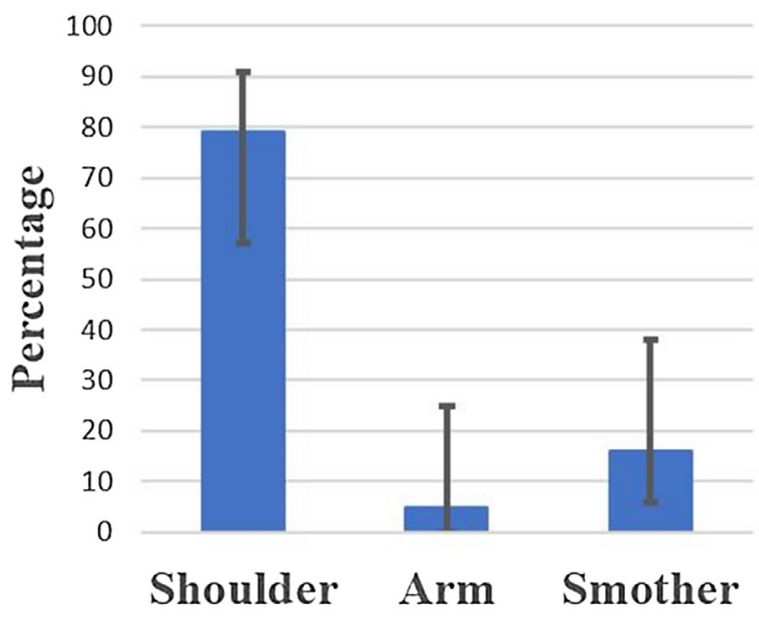

LBT Front-on

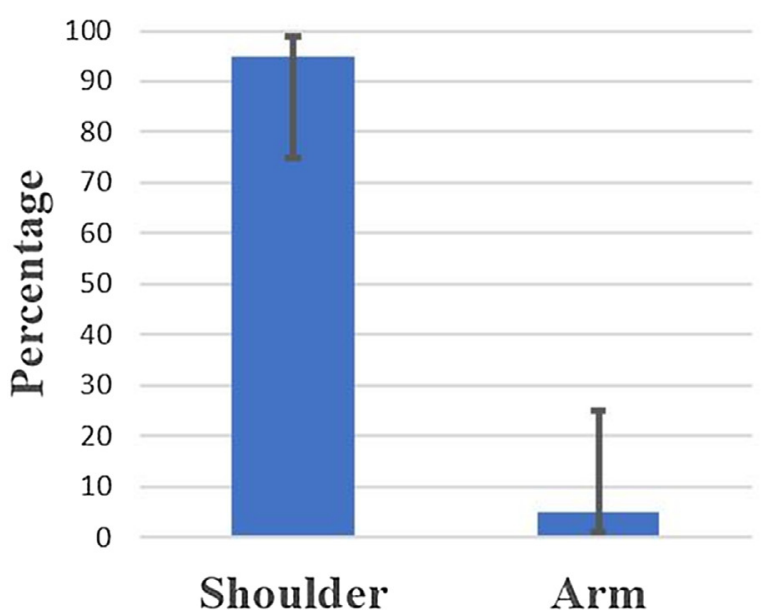

UBT Side-on

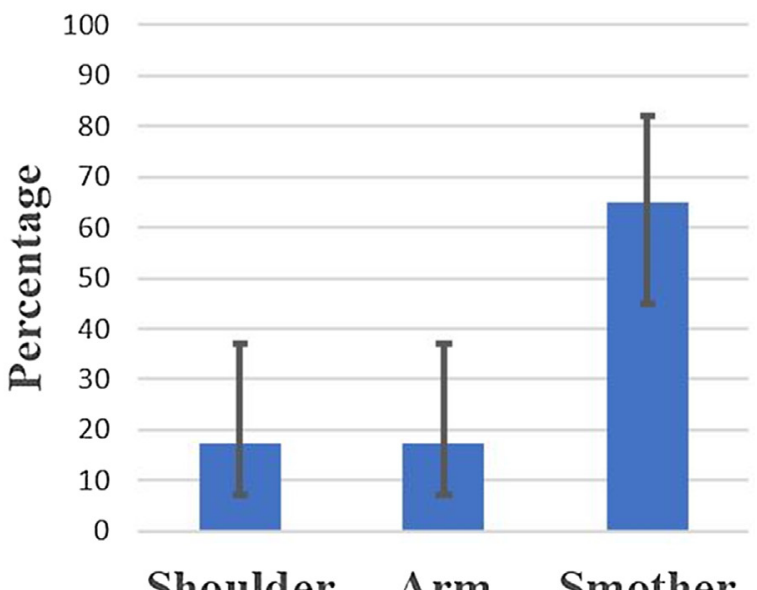

\section{LBT Side-on}

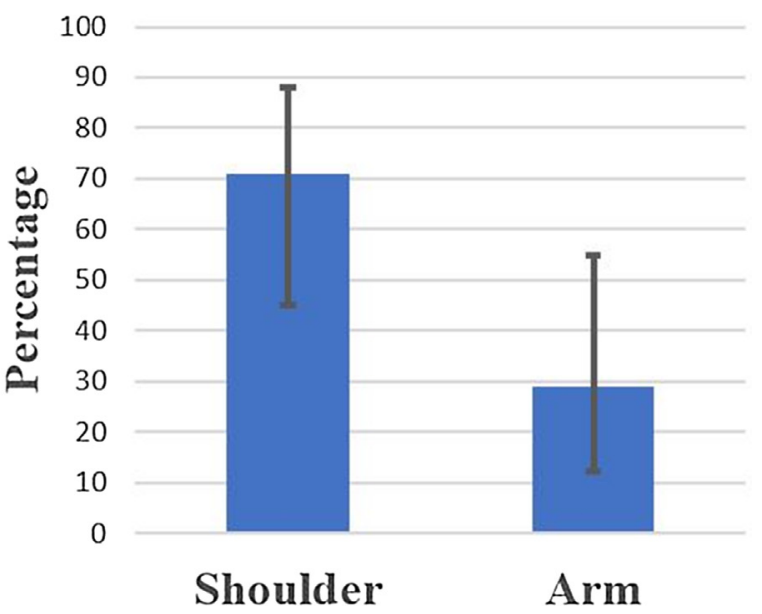

Fig. 2. The distribution of HIA tackles for front- and side-on upper body tackles (UBT) and lower body tackles (LBT) based on tackle type.

$(\mathrm{RR}=2.30 ; 95 \% \mathrm{CI}=1.82-2.92 ; \mathrm{p}<0.01)$. However, intended primary contact at the mid-trunk of the ball carrier had a lower propensity to result in a HIA for the tackler for side-on smother tackles $(\mathrm{RR}=0.11 ; 95 \% \mathrm{CI}=0.02-0.75 ; \mathrm{p}=0.02)$.

Table 1 illustrates that for front-on lower body shoulder tackles, intended primary contact at the lower trunk of the ball carrier had a lower propensity to result in a HIA for the tackler $(R R=0.45$; $95 \% \mathrm{CI}=0.23-0.88 ; \mathrm{p}<0.02$ ). Intended primary contact at the upper leg of the ball carrier had a much greater propensity to result in a HIA for the tackler for front-on $(\mathrm{RR}=2.60 ; 95 \% \mathrm{CI}=1.70-3.97$; $\mathrm{p}<0.01)$ and side-on $(\mathrm{RR}=3.34 ; 95 \% \mathrm{CI}=1.65-6.79 ; \mathrm{p}<0.01)$ lower body shoulder tackles. 
Table 1

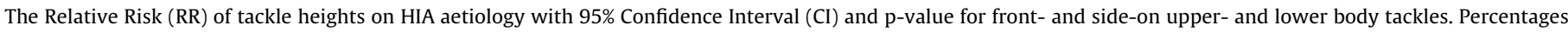
reported include $95 \% \mathrm{CI}$.

\begin{tabular}{|c|c|c|c|c|}
\hline & HIA & Non-HIA & $\mathrm{RR}(95 \% \mathrm{CI})$ & p-value \\
\hline \multicolumn{5}{|l|}{ Upper body tackles } \\
\hline Front-on (Shoulder tackle) & $(n=15)$ & $(n=130)$ & & \\
\hline Upper trunk & 13 (87\%; 62\%-96\%) & 76 (58\%; 50\%-67\%) & $1.48(1.16-1.90)$ & $<0.01$ \\
\hline Mid-trunk & $2(13 \% ; 4 \%-38 \%)$ & $54(42 \% ; 33 \%-50 \%)$ & $0.32(0.09-1.19)$ & 0.09 \\
\hline Side-tn (Smother tackle) & $(\mathrm{n}=15)$ & $(n=148)$ & & \\
\hline Upper trunk & 14 (93\%; 70\%-99\%) & 60 (41\%; 33\%-49\%) & $2.30(1.82-2.92)$ & $<0.01$ \\
\hline Mid-trunk & $1(7 \% ; 1 \%-30 \%)$ & $88(59 \% ; 51 \%-67 \%)$ & $0.11(0.02-0.75)$ & 0.02 \\
\hline \multicolumn{5}{|l|}{ Lower body tackles } \\
\hline Front-on (Shoulder tackle) & $(n=18)$ & $(n=152)$ & & \\
\hline Lower trunk & $6(33 \% ; 16 \%-56 \%)$ & $112(74 \% ; 66 \%-80 \%)$ & $0.45(0.23-0.88)$ & 0.02 \\
\hline Upper leg & 12 (67\%; 44\%-84\%) & 39 (25\%; 19\%-33\%) & $2.60(1.70-3.97)$ & $<0.01$ \\
\hline Lower leg & $0(0 \% ; 0 \%-18 \%)$ & $1(1 \% ; 0 \%-4 \%)$ & $2.68(0.11-63.6)$ & 0.54 \\
\hline Side-on (Shoulder tackle) & $(n=10)$ & $(n=43)$ & & \\
\hline Lower trunk & $3(30 \% ; 11 \%-60 \%)$ & 33 (77\%; 62\%-87\%) & $0.39(0.15-1.02)$ & 0.06 \\
\hline Upper leg & 7 (70\%; 40\%-89\%) & $9(21 \% ; 11 \%-35 \%)$ & $3.34(1.65-6.79)$ & $<0.01$ \\
\hline Lower leg & $0(0 \% ; 0 \%-28 \%)$ & $1(2 \% ; 0 \%-12 \%)$ & $1.33(0.06-30.6)$ & 0.86 \\
\hline
\end{tabular}

Bold values indicate $\mathrm{p}$ values less than 0.05 .

\section{Discussion}

The aim of this study was to use match video evidence of tackles in elite level rugby union to examine the effect of tackle heights on tackler HIA risk for both upper and lower body tackles. The results suggest avoiding the upper trunk for upper body tackles. For lower body tackles, the results suggest tackling at the lower trunk and avoiding the upper legs. These findings can be utilised by coaches to develop tackle height specific prevention strategies and training drills.

Tierney et al. ${ }^{24}$ found that tackles to the upper trunk accounted for almost half (46\%) of all tackle related direct head impacts to the tackler, however, that study did not assess tackle height risk. It has been previously reported that the head and shoulders of the ball carrier were the main body parts to strike the tackler's head in direct head impact ${ }^{2}$ and HIA-related ${ }^{25}$ upper body tackles. This is consistent with the findings of this study as tackling the upper trunk puts the tackler's head closer to these impacting body parts. Furthermore, Tucker et al. ${ }^{25}$ found that an upright tackler, which corresponds to a higher tackle height, was the most common body position to result in a HIA for the tackler. In this study, it was found that in certain upper trunk tackles, the ball carrier entered the tackle in a crouched position meaning that an upper trunk tackle was almost unavoidable, particularly for front-on tackles. Therefore, if a change to the tackle height law was to be made, it would have to ensure that this is mitigated against.

The results of this study indicate that tackling at the upper legs has a higher propensity to result in a HIA than tackling at the upper trunk. Lowering the maximum legal tackle height to below the upper trunk of the ball carrier could reduce HIA risk during upper body tackles. However, it may increase the likelihood of upper leg related HIAs as lowering the tackle height law could increase the number of tackles to the upper leg region. This could have an adverse effect on HIA reduction. Therefore, effective coaching strategies that place emphasis on tackling lower risk body regions such as the mid- and lower trunk are paramount.

The intended primary contact at the upper legs for both frontand side-on lower body tackles showed a greater propensity to result in a HIA for the tackler. The judgement made by the tackler arises in a dynamic situation in which the ball carrier can adjust both running speed and direction. Part of the skill of the ball carrier is to be unpredictable ensuring that the tackler does not make an effective tackle. Therefore, the upper legs of the ball carrier can be moving rapidly which could increase the risk of a head impact for the tackler in comparison to the lower trunk, for example, which reflects the bulk movement of the player. The results also demonstrate that tackling the mid/lower trunk of the ball carrier has a lower propensity to result in a HIA. This supports the recommendation of contacting the ball carrier in the centre of gravity proposed in previous contact technique based studies. ${ }^{9,26}$ Quarrie and Hopkins ${ }^{1}$ found that tackling high (roughly at the upper trunk) was the main legal tackle to cause general injury for the ball carrier in terms of injury rate (3.4 injuries per 1000 tackle events). However, they also found that tackling low (roughly at the upper and lower legs) was the main cause of general injury for the tackler in terms of injury rate (2.2 injuries per 1000 tackle events).

The tackle is a dynamic and open phase of play by nature and this must be considered when analysing tackling. ${ }^{9}$ This is a limitation of technical video analysis. In some tackle scenarios, tackles were executed because of a defensive system error. The tackler may have been forced to execute a tackle as a result of a teammate's missed tackle or poor positioning in the defensive line. In these circumstances, the tackler may not have optimally identified the ball carrier in a timely fashion as their attention was focused on another opposing player. This may have prevented them from reacting to the ball carrier's motion and executing a tackle at the intended tackle height. This highlights the importance of on-field communication and clearly defined defensive systems where players have defined roles and responsibilities. Future work should analyse this aspect of the game.

This study utilised an inclusion criterion based on a player being removed for a HIA and not returning to play for the remainder of the game. This could be considered a strong indication of concussion, however, it cannot be fully regarded as a concussion diagnosis. Access to player medical data would have clarified this. This study utilised elite level rugby union games however further research is required to apply these results towards HIA prevention in both youth and amateur level rugby union. To fully understand the concussion injury mechanism further work must study the biomechanics of rugby union head impacts and tackles. This can be achieved by utilising wearable head sensors or model-based approaches. ${ }^{27,28}$ The approach undertaken in this study focused on the tackler as previous literature has reported that the tackler is at most risk of a head impact, ${ }^{2} \mathrm{HIA}^{25,29}$ and concussion ${ }^{30}$. However, Cross et al. ${ }^{30}$ reported that in $30 \%$ of cases, it is the ball carrier who sustains a concussion. Furthermore, Tucker et al. ${ }^{29}$ reported 0.54 HIAs per 1000 tackles for the ball carrier in a study with a large HIA sample size (464 tackle-related HIAs). Therefore, further work should assess the effect of tackle height on ball carrier HIA risk. Also, 
it is possible that lowering the tackle height law could increase the risk of general injury to players. This would need to be monitored.

Only tackle height was analysed in this study. Modelling the combination and interaction of other technical characteristics and match situation characteristics, such as tackle speed, ${ }^{30}$ could allow for an even greater understanding of HIA risk. Although the HIA sample size was larger than the injury sample size utilised by Burger et al., ${ }^{9}$ the study would have benefited from a larger HIA sample size. The study could be considered underpowered due to the small sample size. This should be considered when interpreting the results. This is a limitation of the data collection approach utilised in this study. Access to an open source video database of head impact/HIA/concussion events would have greatly benefited this study.

\section{Conclusion}

Analysis of match video evidence from elite level rugby union games shows that tackle heights can influence HIA aetiology for the tackler. For front-on upper body shoulder tackles and side-on upper body smother tackles, intended primary contact at the upper trunk of the ball carrier had a greater propensity to result in a HIA for the tackler. However, for side-on upper body smother tackles, intended primary contact at the mid-trunk of the ball carrier had a lower propensity to result in a HIA for the tackler. For front- and side-on lower body shoulder tackles, intended primary contact at the upper leg of the ball carrier had a greater propensity to result in a HIA for the tackler. However, for side-on tackles, intended primary contact at the lower trunk had a lower propensity to result in a HIA for the tackler. To reduce tackler HIA risk, the results suggest tackling below the upper trunk for upper body tackles. The results also support tackling at the lower trunk for lower body tackles and avoiding the upper legs. These findings can be utilised to develop tackle height specific coaching strategies and training drills that place emphasis on tackling lower HIA risk body regions such as the mid- and lower trunk.

\section{Practical implications}

- Lowering the tackle height law to below the upper trunk of the ball carrier could reduce the HIA risk during upper body tackles. However, lowering the tackle height law may increase the likelihood of upper leg related HIAs.

- The results of this study suggest that tackling at the upper legs has a higher propensity to result in a HIA than tackling at the upper trunk. Lowering the tackle height law could subsequently increase the number of tackles to the upper leg region. This could have an adverse effect on HIA reduction.

- The results suggest tackling below the upper trunk for upper body tackles and tackling at the lower trunk for lower body tackles to reduce HIA risk.

- These findings can be utilised to develop tackle height specific coaching strategies and training drills that place emphasis on tackling lower HIA risk body regions such as the mid- and lower trunk.

\section{Acknowledgement}

The authors would like to thank the Irish Research Council for funding this study.

\section{References}

1. Quarrie KL, Hopkins WG. Tackle injuries in professional rugby union. Am J Sports Med 2008; 36(9):1705-1716. http://dx.doi.org/10.1177/0363546508316768.
2. Tierney GJ, Lawler J, Denvir K et al. Risks associated with significant head impact events in elite rugby union. Brain Inj 2016; 30(11):1350-1361. http://dx.doi.org/10.1080/02699052.2016.1193630.

3. Rugby Football Union. England Professional Rugby Injury Surveillance Project 2016-17. Available at: http://www.englandrugby.com/mm/Document/ General/General/01/32/91/95/InjurySurveillanceReport2016-17_English.pdf. Accessed 17 April 2018

4. Brooks JHM, Fuller CW, Kemp SPT et al. Epidemiology of injuries in English professional rugby union: part 1 match injuries. Br J Sports Med 2005; 39(10):757-766. http://dx.doi.org/10.1136/bjsm.2005.018135.

5. Fuller CW, Taylor A, Raftery M. Epidemiology of concussion in men's elite Rugby-7s (Sevens World Series) and Rugby-15s (Rugby World Cup, Junior World Championship and Rugby Trophy, Pacific Nations Cup and English Premiership). Br J Sports Med 2015; 49(7):478-483.

6. Hendricks S, Lambert M. Theoretical model describing the relationship between the number of tackles in which a player engages, tackle injury risk and tackle performance. J Sports Sci Med 2014; 13(3):715.

7. Tierney GJ, Denvir K, Farrell G et al. Does player time-in-game affect tackle technique in elite level rugby union? J Sci Med Sport 2017:1-5. http://dx.doi.org/10.1016/j.jsams.2017.06.023.

8. Tierney GJ, Denvir K, Farrell G et al. The effect of technique on tackle gainline success outcomes in elite level rugby union. Int J Sports Sci Coach 2017:1-10. http://dx.doi.org/10.1177/1747954117711866.

9. Burger N, Lambert MI, Viljoen W et al. Tackle technique and tackle-related injuries in high-level South African Rugby Union under-18 players: real-match video analysis. Br J Sports Med 2016; 50(15):932-938.

10. Hendricks S, O'Connor S, Lambert $\mathrm{M}$ et al. Contact technique and concussions in the South African under-18 Coca-Cola Craven Week Rugby tournament. Eur J Sport Sci 2015; 15(6):557-564.

11. Tierney GJ, Denvir K, Farrell G et al. The effect of tackler technique on head injury assessment risk in elite rugby union. Med Sci Sports Exerc 2017; 50(3):603-608. http://dx.doi.org/10.1249/MSS.0000000000001461.

12. Hendricks S, O'Connor S, Lambert M et al. Video analysis of concussion injury mechanism in under-18 rugby. BMJ Open Sport Exerc Med 2016; 2(1):e000053.

13. Tierney GJ, Simms CK. The effects of tackle height on inertial loading of the head and neck in Rugby Union: a multibody model analysis. Brain Inj 2017:1-7. http://dx.doi.org/10.1080/02699052.2017.1385853.

14. Fuller CW, Ashton T, Brooks JH et al. Injury risks associated with tackling in rugby union. Br J Sports Med 2010; 44(3):159-167.

15. Scher A. The high rugby tackle-an avoidable cause of cervical spinal injury? Drug Res 1978; 5:197

16. Fuller GW, Kemp SPT, Raftery M. The accuracy and reproducibility of video assessment in the pitch-side management of concussion in elite rugby. J Sci Med Sport 2017; 20(3):246-249.

17. McCrory P, Johnston K, Meeuwisse W et al. Summary and agreement statement of the 2nd international conference on concussion in sport, Prague 2004. Br J Sports Med 2005; 39(4):196-204.

18. Tierney GJ, Krosshaug T, Wilson F et al. An assessment of a novel approach for determining the player kinematics in elite rugby union players, IRCOBI Conference Proceedings, 2015.

19. Montgomery C, Blackburn J, Withers D et al. Mechanisms of ACL injury in professional rugby union: a systematic video analysis of 36 cases. Br J Sports Med 2016. http://dx.doi.org/10.1136/bjsports-2016-096425.

20. Tierney GJ, Simms CK. Can tackle height influence tackle gainline success outcomes in elite level rugby union? Int J Sports Sci Coach 2017:1-6. http://dx.doi.org/10.1177/1747954117751345.

21. Landis JR, Koch GG. The measurement of observer agreement for categorical data. Biometrics 1977; 15:9-74.

22. Wilson EB. Probable inference, the law of succession, and statistical inference. $J$ Am Stat Assoc 1927; 22(158):209-212.

23. Hopkins WG. Linear models and effect magnitudes for research, clinical and practical applications. Sportscience 2010; 14(1):49-57.

24. Tierney GJ, Simms CK. The effect of intended primary contact location on tackler head impact risk, IRCOBI Conference Proceedings, 2017.

25. Tucker R, Raftery M, Kemp S et al. Risk factors for head injury events in professional rugby union: a video analysis of 464 head injury events to inform proposed injury prevention strategies. Br J Sports Med 2017; 51(15):1152-1157.

26. Gabbett T, Kelly J. Does fast defensive line speed influence tackling proficiency in collision sport athletes? Int J Sports Sci Coach 2007; 2(4):467-472.

27. Tierney GJ, Joodaki H, Krosshaug T et al. The kinematics of head impacts in contact sport: an initial assessment of the potential of model based image matching, ISBS-Conference Proceedings Archive, 2016.

28. Tierney GJ, Joodaki H, Krosshaug $\mathrm{T}$ et al. Assessment of modelbased image-matching for future reconstruction of unhelmeted sport head impact kinematics. Sports Biomech 2017:1-15. http://dx.doi.org/10.1080/14763141.2016.1271905.

29. Tucker R, Raftery M, Fuller GW et al. A video analysis of head injuries satisfying the criteria for a head injury assessment in professional rugby union: a prospective cohort study. Br J Sports Med 2017; 51(15):1147-1151.

30. Cross MJ, Tucker R, Raftery $M$ et al. Tackling concussion in professional rugby union: a case-control study of tackle-based risk factors and recommendations for primary prevention. Br J Sports Med 2017:1-6. http://dx.doi.org/10.1136/bjsports-2017-097912. 